\title{
Cuarentena por COVID-19, factor asociado al consumo de drogas legales en estudiantes de enfermería
}

\author{
Est. Carlos Enrique Fernández Hernández \\ Dr. Javier Salazar Mendoza \\ M.C. Enf. María Esperanza Conzatti Hernández \\ Dra. Edith Castellanos Contreras \\ M.C. Enf. María de los Ángeles Onofre Santiago \\ LE. Oscar Daniel Luna Hernández. \\ Dra. Ivett Rodríguez Muñoz \\ Dra. Ernestina Méndez Cordero \\ Universidad Veracruzana, Mexico
}

Doi:10.19044/esj.2021.v17n10p1

Submitted: 11 February 2021

Accepted: 12 March 2021

Published: 31 March 2021
Copyright 2021 Author(s)

Under Creative Commons BY-NC-ND

4.0 OPEN ACCESS

Cite As:

Fernández Hernández C.E., Salazar Mendoza J., Conzatti Hernández M.E., Castellanos Contreras E., Onofre Santiago M.L., Luna Hernández O.D., Rodríguez Muñoz I. \& Méndez Cordero E. (2021). Cuarentena por COVID-19, factor asociado al consumo de drogas legales en estudiantes de enfermería. European Scientific Journal, ESJ, 17(10), 1.

https://doi.org/10.19044/esj.2021.v17n10p1

\section{Resumen}

Introducción: en la pandemia por COVID-19 se han implementado políticas de salud poco usuales como la cuarentena en la población, modificando entornos sociales, laborales y escolares siendo los jóvenes los más afectados ya que no todos estaban preparados para acatar esas indicaciones, generando estrés o ansiedad, afecciones que generalmente son remediadas por el consumo de drogas psicoactivas como alcohol y tabaco. Objetivo: determinar si la cuarentena por COVID-19, es un factor de consumo de drogas legales en estudiantes de Enfermería. Método: investigación cuantitativa, descriptiva, correlacional, prospectiva y trasversal, en una muestra de 266 estudiantes de la Licenciatura de Enfermería de la Región Orizaba de la Universidad Veracruzana, con muestreo no probabilístico por cuota. Se aplicó el AUDIT y ASSIST de la OMS. Resultados: $86.1 \%$ posee conocimiento alto sobre COVID-19, 63.5\% ingirió alcohol en la vida, 30.8\% toma de 1 a 2 copas y $93.3 \%$ nunca perdió la noción de lo que sucedía. Por 
otro lado, $59.0 \%$ probó tabaco, $16.2 \%$ consumieron 1 o 2 veces en tres meses y $100 \%$ negó padecer problemas por este hábito. Conclusión: $20.4 \%$ son hombres y $79.6 \%$ mujeres de distintas edades y semestres, $92.5 \%$ no tuvo COVID-19, 53.0\% son consumidores ocasionales de alcohol y $29.5 \%$ fuman con riesgo bajo, en aquellas personas que tuvieron resultados bajos de conocimiento de COVID-19, aumentó el uso de alcohol ( $r s=-.121, \mathrm{p}<0.05$ ), para el uso de tabaco no hubo cambios; los resultados se contraponen con otros autores.

Palabras claves: Pandemia, COVID-19, cuarentena, drogas y estudiantes de Enfermería

\section{COVID-19 Quarantine, a Factor Associated with Legal Drug Use in Nursing Students}

Est. Carlos Enrique Fernández Hernández

Dr. Javier Salazar Mendoza

M.C. Enf. María Esperanza Conzatti Hernández

Dra. Edith Castellanos Contreras

M.C. Enf. María de los Ángeles Onofre Santiago

LE. Oscar Daniel Luna Hernández

Dra. Ivett Rodríguez Muñoz

Dra. Ernestina Méndez Cordero

Universidad Veracruzana, Mexico

\section{Abstract}

Introduction: during the COVID-19 pandemic, unusual health policies such as quarantine have been implemented in the population, modifying social, work and school environments, with young people being the most affected since not all of them were prepared to comply with these indications, generating stress or anxiety, conditions that are generally remedied by the consumption of psychoactive drugs such as alcohol and tobacco. Objective: to determine whether COVID-19 quarantine is a factor in the consumption of legal drugs in nursing students. Method: quantitative, descriptive, correlational, prospective and transversal research, in a sample of 266 students of the Nursing Degree of the Orizaba Region of the Universidad Veracruzana, with non-probabilistic sampling by quota. The AUDIT and ASSIST of the WHO were applied. Results: $86.1 \%$ had a high knowledge of COVID-19, 63.5\% had ingested alcohol in their lifetime, $30.8 \%$ had 1 to 2 drinks and $93.3 \%$ had never lost track of what was happening. On the other hand, $59.0 \%$ tried tobacco, $16.2 \%$ consumed 1 or 2 times in three months and 
$100 \%$ denied suffering problems due to this habit. Conclusion: $20.4 \%$ are men and $79.6 \%$ women of different ages and semesters, $92.5 \%$ did not have COVID-19, 53.0\% are occasional alcohol consumers and 29.5\% smoke with low risk, in those people who had low COVID-19 knowledge results, alcohol use increased ( $r s=-.121, p<0.05)$, for tobacco use there were no changes; the results are in contrast with other authors.

Keywords: Pandemic, COVID-19, quarantine, drugs and nursing students

\section{Introduction}

La pandemia de COVID-19 derivada de la enfermedad por el nuevo virus SARS-COV-2 que se identificó el 31 de diciembre de 2019 en una ciudad asiática, afectó de manera negativa a los individuos de diferentes naciones (BBC News, 2020a), pues para el 27 de febrero de 2020, se habían confirmado 82 mil 294 casos de infección en todo el mundo, de los cuales $95.5 \%$ se concentraba en China y el porcentaje restante permanecía disperso en 57 países más, donde Estados Unidos, Canadá y Brasil eran las únicas naciones del continente americano con casos confirmados (Secretaría de Salud, 2020).

La enfermedad por coronavirus (COVID-19) es de tipo infecciosa causada por un coronavirus con cuadros respiratorios de leves a moderados, con afecciones médicas subyacentes, como enfermedades cardiovasculares, diabetes, enfermedades respiratorias crónicas o cáncer, tienen más probabilidades de presentar un cuadro grave, se propaga a través de las gotículas de saliva o las secreciones nasales que se generan cuando una persona infectada tose o estornuda (OMS, 2021), al tratarse de un problema de salud nuevo, provocó que se dispersara información verdadera y falsa por diferentes medios de comunicación de una manera masiva, pues el hecho de enfrentarla era importante ya que se había convertido rápidamente en una pandemia, generó que se volviera el foco de atención de la humanidad sin importar el grupo de edad al que perteneciera (OPS, 2020a).

Los diferentes Gobiernos, a fin de limitar el contacto y expansión de la enfermedad, estipularon medidas de distanciamiento y fue la Organización Mundial de la Salud (2020c), quien estipuló el período de cuarentena por COVID-19 que se refiere a restringir actividades o separar a las personas que no están enfermas pero que pueden haber estado expuestas al SARS-CoV-2, con el objetivo de prevenir la propagación de la enfermedad en el momento en que los usuarios empiezan a presentar síntomas o no.

En México, aún cuando se respetó el distanciamiento social, la actividad de las personas continuó con nuevas formas y con ello el acceso instantáneo a todo tipo de información a través del internet, la facilidad de conocer las cifras estadísticas en cualquier momento, los cambios económicos y grandes restricciones públicas afectan a la representación de una nación 
como a su población generando y agraviando problemas psicológicos como estrés o ansiedad, siendo los jóvenes más afectados, pues es muy común que ellos quieran remediar ese mal con alguna sustancia que altere su estado de conciencia (Oteo, 2005), en muchos de los casos, drogas legales que son aquellas sustancias con efectos psicoactivos permitidas, no siendo su uso penado por ley a pesar de conocerse sus posibles efectos. Este estatus legal puede referirse a su uso a nivel recreativo o bien a estar pensados para otros usos, que no incluyen su consumo para lograr alterar la psique, y por los cuales se considera algo permitido como el alcohol y tabaco (Psicología y mente, 2021).

Es por eso, que las personas se encuentran inmersas en una sociedad de cambio constante donde el consumo de drogas influye de manera masiva (Oteo, 2005). No obstante, la situación actual se caracteriza por políticas de salud estrictas e implementadas forzosamente en todo el mundo y han modificado completamente el ámbito social, laboral, cultural e incluso escolar de los jóvenes (BBC News, 2020b). En un informe realizado por Sun et al. (2020) a 6 mil 416 personas en China sobre su comportamiento adictivo a internet y sustancias durante la pandemia de COVID-19, los resultados obtenidos mencionan que $46.8 \%$ tuvo mayor dependencia al uso de internet, $32.7 \%$ de bebedores de alcohol y $13.6 \%$ de fumadores aumentaron la cantidad de consumo.

De igual forma, a nivel nacional (Natera et al., 2020), realizaron un estudio a 679 mexicanos para evaluar la implementación de un programa preventivo sobre el consumo de alcohol durante la pandemia, encontrando que el $65.4 \%$ presentó ingesta moderada de bebidas embriagantes, $2.2 \%$ son consumidores de riesgo y al menos $30 \%$ mantuvo abstinencia a esta droga durante marzo y agosto de 2020. Por lo tanto, el devastador efecto que viene ocasionando esta pandemia como la propagación y generalización de la enfermedad, han originado que se dicten políticas gubernamentales de salud pública, que se enfocan principalmente en el distanciamiento social y cuarentena de la población (CDC, 2020), cuyo objetivo general es que todos los países controlen la afección, frenando la transmisión y reduciendo la mortalidad asociada a la enfermedad (OMS, 2020a).

Sin embargo, no todas las personas estaban preparadas para acatar esas indicaciones sobre todo en los hogares donde el ingreso económico mensual es mínimo, ya que la mayoría de la población económicamente activa, genera ganancias al día y por otra parte los estudiantes de diferentes niveles no contaban con el recurso tecnológico o de red para cumplir con sus actividades académicas, generando preocupación, estrés, ansiedad y en algunos casos, exposición al consumo de drogas legales como alcohol y tabaco (OPS, 2020b). A nivel nacional, la Comisión Nacional Contra las Adicciones (CONADIC), menciona que el consumo de drogas lícitas (alcohol y tabaco) se ha ubicado 
como uno de los problemas de salud pública de mayor relevancia, debido a las consecuencias sanitarias y sociales que experimentan quienes las consumen (CONADIC, 2017).

El beber alcohol sigue obteniendo datos elevados pues de acuerdo con la Encuesta Nacional de Consumo de Drogas, Alcohol y Tabaco (ENCODAT), reporte de alcohol 2016-2017 elaborado por la Secretaría de Salud, la prevalencia de consumo de bebidas embriagantes en 2016 fue de $53.3 \%$, así como la ingesta en el último mes de 39.9\% para la población adulta de 18 a 65 años (Secretaría de Salud, 2017a). En cuanto al consumo de tabaco, 14.9 millones de mexicanos entre 12 a 65 años lo fumaba, siendo los hombres lo de mayor proporción (11 millones 78 mil) que las mujeres ( 3 millones 812 mil) (Secretaría de Salud, 2017b). Por lo antes citado y la búsqueda de información en diversas fuentes, se confirma la importancia del estudio: si la cuarentena por COVID-19 es un factor predisponente para el consumo de drogas legales en estudiantes de Enfermería es relevante, puesto que en la actualidad no existe suficiente información que indague sobre el hábito de ingesta de alcohol y tabaco en la población durante el confinamiento, debido a la escasez de estudios que entrelacen estos factores, siendo la principal limitante al realizar este proyecto, independientemente de la dificultad para adaptarse a una nueva normalidad.

\section{Objetivo}

Determinar si la cuarentena por COVID-19, es un factor de consumo de drogas legales en estudiantes de Enfermería.

\section{Método}

El diseño de la presente investigación por el análisis y alcance de resultados es cuantitativa, de diseño descriptiva y correlacional, exponiendo los resultados de forma numérica y describiendo los hallazgos más importantes a fin de contrastar con hechos empíricos; por el tiempo de ocurrencia de los acontecimientos y registro de información recabando los datos de la fuente directa; por la secuencia del estudio durante el período septiembre 2020-febrero 2021 es transversal, determinando la cuarentena por COVID-19 como factor asociado al consumo de drogas legales en estudiantes de Enfermería y mediante la aplicación, se dio respuesta a los objetivos planteados (Canales, Alvarado \& Pineda, 2013; Ortiz \& García 2014; Grove, Gray \& Burns, 2016; Hernández, Fernández \& Baptista, 2014).

\section{Muestra}

El estudio se llevó a cabo en la Facultad de Enfermería de la Región Orizaba perteneciente a la Universidad Veracruzana, con estudiantes de tercero, quinto, séptimo, noveno, onceavo y treceavo semestre, así como 
pasantes en servicio social de la Licenciatura en Enfermería del plan de estudios 2013, obteniendo un total de 540 personas.

De acuerdo con Polit y Hungler (2020), aplicando la fórmula para poblaciones finitas, con un nivel de significancia estadística de 1.96 (95.0\%) y error máximo aceptable de 0.05 , se obtuvo una muestra de 225 casos, representando el $41.66 \%$ de la población; por la temática abordada se determinó aplicar el instrumento de investigación a un total final de 266 estudiantes (49.0\%) de la Licenciatura de Enfermería de la Universidad Veracruzana, en el período septiembre 2020-febrero 2021.

\section{Muestreo}

La obtención de la muestra fue a través del método de muestreo no probabilístico por cuota que, según Canales, Alvarado y Pineda (2013), consiste en que el investigador selecciona la muestra considerando el fenómeno o variables a estudiar. Para este caso fue que los participantes estuvieran inscritos en la Facultad de Enfermería Orizaba de la Universidad Veracruzana, cursando el tercer período o superior, sin importar el sexo, edad o religión y retomando el Concentrado de inscripción de la Facultad de Enfermería Orizaba, período septiembre 2020-febrero 2021.

Por otra parte, los criterios de selección fueron, inclusión: estudiantes de la Licenciatura de Enfermería inscritos en el período septiembre 2020febrero 2021 de la Facultad de Enfermería Orizaba, de tercero, quinto, séptimo, noveno, onceavo, treceavo o más semestres con rango de edad entre 18 y 30 años, sexo indistinto, con disponibilidad de tiempo, que estuvieron dispuestos a participar en dicha investigación, que accedieron a responder el instrumento en versión electrónica y que firmaron el consentimiento informado (Google, 2020b). Se excluyeron a los alumnos de nuevo ingreso, así como a los casos que presentaron tratamiento para dejar de consumir alcohol y tabaco. Se eliminaron a los participantes que costearon dos o más veces, no completaron el instrumento aplicado, mostraron desinterés o poca confiabilidad en sus respuestas, que desertaron al momento de responder el cuestionario y los casos que tuvieran una matrícula diferente a las registradas en el concentrado de inscripción del período septiembre 2020-febrero 2021 (Tamayo, 2014).

\section{Instrumento de medición}

Se diseñó de manera electrónica en la plataforma de Google formularios (Google, 2020b), acompañado de un formato de consentimiento informado aprobado por el entrevistado, con los nombres del entrevistador y director del proyecto, también se integró una cédula de identificación de datos, que permitió evaluar las características sociodemográficas de la muestra en estudio, conteniendo edad, sexo, estado civil, lugar de procedencia, práctica 
religiosa, con quién vive el estudiante e incluso dos preguntas que hacen referencia al diagnósticos y cuarentena que experimentaron los usuarios durante el período septiembre 2020-febrero 2021.

En el segundo apartado se encuentra el cuestionario sobre conocimiento general de COVID-19 y medidas de prevención, retomado por las recomendaciones de la Organización Mundial de la Salud (2020b). Está Integrado por 16 reactivos con respuestas de opción múltiple, evalúa conocimientos y generalidades sobre la nueva enfermedad, preguntando a los estudiantes las medidas de higiene y protección, sana distancia, identificación de síntomas y estrategias de afrontamiento (Google, 2020b).

El tercer apartado está dirigido para medir la variable consumo de alcohol con el instrumento: Identificación de Desórdenes por Uso de Alcohol (AUDIT, por sus siglas en inglés), que fue desarrollado por la Organización Mundial de la Salud (2001) junto a un grupo internacional de investigadores, publicado en 1989 y actualizado en 1992 por Babor, Higgins, Suander \& Monteiro (2001), por otro lado, Garcúa, Novalbos, Martínez \& O’Ferral (2016), mencionan en un estudio de validación que la fiabilidad y validez del test fue aceptable, puesto que utilizaron el alfa de Cronbach, obteniendo 0.75 y demostrando una buena consistencia interna. Este instrumento está integrado por 10 preguntas: las tres primeras hacen referencia a la frecuencia y cantidad sobre el consumo de alcohol, las tres siguientes evalúan los síntomas de dependencia al producto y las últimas cuatro, investigan los resultados obtenidos por este hábito. Cada una de las preguntas tiene una escala de respuesta de 0 a 4 , dando una puntuación máxima de 40 y mínima 0 . Los resultados se clasifican en 0 abstinencia, 1 y 7 consumo ocasional, 8 a 15 consumo de riesgo, 16 y 19 consumo perjudicial e igual o mayor a 20 dependencia al alcohol.

En el cuarto apartado está dirigido a la evaluación de consumo de tabaco mediante la Prueba de Detección de Consumo de Alcohol, Tabaco y Sustancias (ASSIST, por sus siglas en inglés), el cual fue desarrollado por la Organización Mundial de la Salud en 1997 (OMS, 2004) y adecuado a la población latinoamericana por la Organización Panamericana de la Salud en 2011 y Tiburcio et al. (2016), mencionan en su estudio titulado Validez y confiabilidad de la prueba de detección de consumo de tabaco y sustancias (ASSIST) en estudiantes universitarios aplicado a 1176 estudiantes mexicanos que dicho cuestionario obtuvo 0.87 de fiabilidad general mediante la prueba de consistencia Alfa de Cronbach, así como una puntuación de 0.83 en la misma prueba de consistencia, pero solo en la variable tabaco.

Las opciones de respuestas del instrumento son las siguientes: la primera pregunta identifica el consumo de cualquier sustancia en algún momento de la vida del entrevistado, con opciones de respuesta dicotómica (sí y no) con una puntuación de 3 y 0 respectivamente. Las demás interrogantes 
hasta la séptima muestran respuestas de tipo Likert. Al final de la entrevista se suman las puntuaciones de las preguntas 1 a la 7 para cada sustancia específica. Se debe hacer una sumatoria, generando un puntaje mínimo de 0 y máximo de 39. Se consideran de riesgo bajo a aquellos puntuados entre 0 y 3 , riesgo moderado los de 4 a 26 y riesgo alto para más quienes acumulan más de 27 puntos.

\section{Recolección y análisis de datos}

El proyecto fue autorizado por el comité de bioética de la Facultad de Enfermería de Orizaba, previa solicitud por parte de los responsables, después, se asignó un Director del proyecto donde se expuso el objetivo del estudio y finalidad. Posteriormente, se procedió a trabajar con los estudiantes de la Licenciatura en Enfermería de los diferentes semestres del período escolar septiembre 2020-febrero 2021, de la Región Orizaba, utilizando la técnica de entrevista y el método, un instrumento previamente seleccionado, que cubriera los criterios de fiabilidad, validez y consistencia interna, mismo que se adaptó a su versión electrónica (Google, 2020b) para facilitar la obtención de información y proceder al análisis.

\section{Análisis de datos}

Se utilizó el programa Google Formularios (Google, 2020a) para convertir el instrumento de medición en formato digital y así elaborar un análisis de la información mediante la creación de una base de datos en Excel. Dichos resultados, se transformaron a códigos numéricos para transferirlos al programa digital Statistical Package for the Social Sciences (SPSS) versión 15.0.1 para Windows (SPSS, Inc. 2006), formando una nueva base de datos donde se integran todas las derivaciones obtenidas de los instrumentos aplicados previa validación y revisión. El plan de análisis estuvo integrado por estadística descriptiva (Celis \& Labrada, 2014; Arceo, Ornelas \& Domínguez, 2010; Orellana, 2001), mediante el uso de frecuencia, porcentajes, medidas de tendencia central (media, mediana, moda) y dispersión (desviación típica, mínimo y máximo); para evaluar la normalidad de las variables, se utilizó la prueba de Kolmogorov Smirnov (García, González, Jornet, 2010), mediante Rho Spearman (Díaz et al., 2014), se determinó la correlación de variables. 


\section{Resultados}

Tabla 1. Generación, semestre, edad y estado civil por sexo

\begin{tabular}{|c|c|c|c|c|}
\hline \multirow{3}{*}{ Generación } & \multicolumn{4}{|c|}{ Sexo } \\
\hline & \multicolumn{2}{|c|}{ Masculino } & \multicolumn{2}{|c|}{ Femenino } \\
\hline & $\mathbf{f}$ & $\%$ & $\mathbf{f}$ & $\%$ \\
\hline 1900 & 14 & 5.3 & 63 & 23.7 \\
\hline 1800 & 10 & 3.8 & 48 & 18.0 \\
\hline 1700 & 20 & 7.5 & 73 & 27.4 \\
\hline 1600 & 8 & 3.0 & 23 & 8.6 \\
\hline 1500 & 1 & 0.4 & 2 & 0.8 \\
\hline Otra & 1 & 0.4 & 3 & 1.1 \\
\hline \multicolumn{5}{|l|}{ Semestre que cursa actualmente } \\
\hline Tercero & 14 & 5.3 & 63 & 23.7 \\
\hline Quinto & 10 & 3.8 & 49 & 18.4 \\
\hline Séptimo & 20 & 7.5 & 73 & 27.4 \\
\hline Noveno & 8 & 3.0 & 23 & 8.6 \\
\hline Onceavo & 1 & 0.4 & 1 & 0.4 \\
\hline Treceavo semestre o más & 1 & 0.4 & 3 & 1.1 \\
\hline \multicolumn{5}{|l|}{ Grupo de edad } \\
\hline 18 a 20 años & 10 & 3.8 & 71 & 26.7 \\
\hline 21 a 23 años & 29 & 10.9 & 115 & 43.2 \\
\hline 24 a 26 años & 10 & 3.8 & 15 & 5.6 \\
\hline 27 a 29 años & 2 & 0.8 & 8 & 3.0 \\
\hline 30 años y más & 3 & 1.1 & 3 & 1.1 \\
\hline \multicolumn{5}{|l|}{ Estado civil } \\
\hline Soltero(a) & 47 & 17.7 & 189 & 71.0 \\
\hline Casado(a) & 2 & 0.8 & 11 & 4.1 \\
\hline Unión libre & 5 & 1.9 & 12 & 4.5 \\
\hline & 54 & $20.4 \%$ & 212 & $79.6 \%$ \\
\hline
\end{tabular}

Nota: Fuente: cédula de identificación de datos, $n=266$.

La Tabla 1, muestra la generación, semestre, grupo de edad y estado civil en relación con el sexo de los estudiantes. La de mayor participación fue de la matrícula 1700 con el $34.9 \%$ ( $7.5 \%$ masculinos y $27.4 \%$ femeninos) y los de menor valor fueron de la 1500 ( $0.4 \%$ masculinos y $0.8 \%$ femeninos). Lo anterior confirma los criterios del Plan de Estudios 2013 de la Licenciatura en Enfermería (Universidad Veracruzana, 2013), donde señala que el tiempo promedio de egreso/titulación es de cinco años, es decir que de la matrícula 1500 , en su mayoría egresaron o se encuentran en proceso de titulación. Por otra parte, la segunda variable expresa que el mayor número de participantes 
cursan el séptimo período, pues se obtuvo un 34.9\% (7.5\% masculinos y $27.4 \%$ femeninos) mientras que los alumnos que cursan el onceavo semestre colaboraron un $0.8 \%$ ( $0.4 \%$ masculinos y $0.4 \%$ femeninos). Algo semejante ocurre con el grupo de edad, ya que sobresalió el rango de 21 a 23 años con un $54.1 \%$ ( $10.9 \%$ masculinos y $43.2 \%$ femeninos) en contraste con el $2.2 \%$ (1.1\% masculinos y $1.2 \%$ femeninos) que obtuvieron los del sector de 30 años y más, relacionando lo planteado en el documento titulado Principales cifras del sistema educativo nacional 2018-2019 (Secretaría de Educación Pública, 2019), el cual señala que existe una prevalencia de adultos jóvenes con 18 a 22 años en educación a nivel superior.

Así mismo, en el estado civil predominaron los participantes solteros de aquellos que están casados, pues se obtuvo el $88.7 \%$ (17.7\% masculinos y $71.0 \%$ femeninos $)$ y $4.9 \% \quad(0.8 \%$ masculinos y $4.1 \%$ femeninos $)$ respectivamente, relacionándose con un estudio (Pineda, Lara, Rocha, Piña \& Romero, 2016) a 469 estudiantes de la Licenciatura en Enfermería en México. Dicha investigación expone que el $97 \%$ de los participantes son solteros ya que el rango promedio de edad fue de 20 , lo que se relaciona a tener un buen rendimiento escolar dado que cuenta con mayor tiempo para las actividades académicas, relaciones interpersonales y concluir con éxito su formación profesional.

Para resumir la variable de sexo, es significativo que en todos los casos sobresalió el femenino pues en las diferentes matrículas, semestre actual, grupo de edad y estado civil se obtuvo el 79.6\% de participación (211 usuarias de la población total final) lo que confirma que en el área de enfermería siguen predominando las mujeres sin embargo, existe aumento del número de varones que solicitan unirse a esta carrera, pues en la actualidad, la Universidad Veracruzana no hace distinción de género en ninguna área (Universidad Veracruzana, 2013).

Tabla 2. Antecedentes de COVID-19

\begin{tabular}{lcccccc}
\hline \multirow{2}{*}{ Antecedente } & \multicolumn{2}{c}{ Sí } & \multicolumn{2}{c}{ No } & \multicolumn{2}{c}{ Total } \\
\cline { 2 - 7 } & f & $\mathbf{\%}$ & f & $\mathbf{\%}$ & f & \% \\
\hline Tiene o tuvo COVID-19 & 20 & 7.5 & 246 & 92.5 & $\mathbf{2 6 6}$ & $\mathbf{1 0 0 \%}$ \\
Estuvo en cuarentena por diagnóstico de COVID-19 & 31 & 11.7 & 235 & 88.3 & $\mathbf{2 6 6}$ & $\mathbf{1 0 0 \%}$ \\
\hline
\end{tabular}

Nota: Fuente: cédula de identificación de datos, $n=266$.

En la Tabla 2, se hace mención sobre los participantes que fueron diagnosticados con COVID-19 así como los que permanecieron resguardados en sus casas por dicha enfermedad. El 7.5\% afirmó haber padecido esta afección en algún momento y el $92.5 \%$ respondió negativamente esta interrogante. Por otro lado, el $11.7 \%$ mencionaron haber estado en cuarentena por probable diagnóstico o diagnóstico confirmado de sars-cov-2 y el $88.3 \%$ 
no, sin embargo, estas personas resguardaron su integridad haciendo caso a las recomendaciones de las diferentes entidades sanitarias.

Lo anterior tiene relación con el comunicado oficial número $174 \mathrm{del}$ Plan COVID-19 (H. Ayuntamiento de Orizaba, 2020), que menciona que para el 31 de agosto del 2020 existían mil 558 casos confirmados acumulados en esa región, 124 personas sospechosas de padecerla y al menos 780 resultados fueron negativos a esta enfermedad.

Tabla 3. Conocimiento del COVID-19

\begin{tabular}{|c|c|c|c|c|}
\hline \multirow{2}{*}{ Conocimiento } & \multicolumn{2}{|c|}{ Correcto } & \multicolumn{2}{|c|}{ Incorrecto } \\
\hline & f & $\%$ & $\mathbf{f}$ & $\%$ \\
\hline $\begin{array}{l}\text { Los coronavirus causan enfermedades respiratorias en humanos y } \\
\text { animales }\end{array}$ & 266 & 100 & 0 & 0 \\
\hline El SARS y MERS-CoV son enfermedades de los coronavirus & 228 & 85.7 & 38 & 14.3 \\
\hline SARS-CoV-2 es el nombre del nuevo coronavirus & 220 & 82.7 & 46 & 17.3 \\
\hline De persona a persona se propaga el nuevo coronavirus & 264 & 99.2 & 2 & 0.8 \\
\hline OVID-19 es el nombre de la nueva enfermedad & 226 & 85.0 & 40 & 15.0 \\
\hline Una persona asintomática puede contagiar de COVID-19 & 236 & 88.7 & 30 & 11.3 \\
\hline Tos, fiebre y cefalea son síntomas principales de COVID-19 & 158 & 59.4 & 108 & 40.6 \\
\hline $\begin{array}{l}\text { Personas con sistema débil o enfermedades empeoran por COVID- } \\
19\end{array}$ & 254 & 95.5 & 12 & 4.5 \\
\hline Debe ponerse en cuarentena si se expuso al COVID-19 & 224 & 84.2 & 42 & 15.8 \\
\hline $\begin{array}{l}\text { La cuarentena por COVID-19 es restringir el movimiento de la } \\
\text { gente }\end{array}$ & 182 & 68.4 & 84 & 31.6 \\
\hline $\begin{array}{l}\text { El aislamiento por COVID-19 es separar a los enfermos de los } \\
\text { demás }\end{array}$ & 216 & 81.2 & 50 & 18.8 \\
\hline $\begin{array}{l}\text { Distanciamiento físico, es alejarse físicamente de otra persona ( } 1.5 \\
\text { metros) }\end{array}$ & 250 & 94.0 & 16 & 6.0 \\
\hline $\begin{array}{l}\text { El cloro es el desinfectante para utilizar en casa y evitar el COVID- } \\
19\end{array}$ & 133 & 50.0 & 133 & 50.0 \\
\hline $\begin{array}{l}\text { El distanciamiento físico y cuarentena previenen el contagio de } \\
\text { COVID-19 }\end{array}$ & 262 & 98.5 & 4 & 1.5 \\
\hline El uso de cubrebocas no evita el contagio de COVID-19 & 28 & 10.5 & 238 & 89.5 \\
\hline Los síntomas del COVID-19 aparecen entre 1 y 12 días & 159 & 59.8 & 107 & 40.2 \\
\hline
\end{tabular}

Nota: Fuente: cédula de identificación de datos, SARS= Síndrome respiratorio agudo severo, MERS-CoV= Síndrome respiratorio de oriente medio, SARS-CoV-2= Síndrome respiratorio agudo severo Coronavirus tipo 2, COVID-19= Enfermedad por coronavirus de $2019, n=266$.

La Tabla 3 demuestra el conocimiento general del COVID-19, así como la detección o aparición de signos y síntomas, factores de riesgo, medidas de prevención o reducción y diferenciación de conceptos como cuarentena, aislamiento y distanciamiento por la nueva enfermedad. Cabe señalar que en la mayoría de las preguntas se obtuvo un valor por encima del 
$80.0 \%$ en respuestas correctas, por ejemplo, la variable de las afecciones que causan los coronavirus presentó $85.7 \%$ e incluso, el $100 \%$ respondió correctamente qué son los coronavirus, es decir que la población conoce e identifica los elementos principales básicos de la enfermedad y cuentan con una formación que permite comprender el problema

Por otro lado, en la variable tiempo en que se desarrollan los síntomas en relación con la exposición del agente patógeno obtuvo un $59.8 \%$ de respuestas acertadas, $50.0 \%$ respondió correctamente la pregunta del desinfectante a utilizar en casa y tan sólo el $10.5 \%$ acertó sobre el uso de cubrebocas, relacionándose con un estudio elaborado en Cuba (Gómez, Diéguez, Pérez, Tamayo \& Iparraguirre, 2020), ya que reportaron que el 95.4\% tenía conocimiento general sobre el COVID-19, el 80.0\% reconocieron los síntomas clínicos de la enfermedad y el $93.7 \%$ conocen la diferenciación de ésta con otras afecciones respiratorias.

Tabla 4. Clasificación de la COVID-19 y consumo de alcohol

\begin{tabular}{lccccccc}
\hline \multirow{2}{*}{ Consumo de alcohol } & \multicolumn{4}{c}{ Conocimiento de COVID-19 } \\
\cline { 2 - 8 } & \multicolumn{2}{c}{ CM } & \multicolumn{3}{c}{ CA } & \multicolumn{2}{c}{ Total } \\
\cline { 2 - 7 } & f & \% & f & \% & f & \% \\
\hline No consume alcohol & 20 & 7.5 & 86 & 32.2 & $\mathbf{1 0 6}$ & $\mathbf{3 9 . 7 \%}$ \\
Consumo ocasional & 14 & 5.3 & 127 & 47.7 & $\mathbf{1 4 1}$ & $\mathbf{5 3 . 0 \%}$ \\
Consumo de riesgo & 1 & 0.4 & 14 & 5.3 & $\mathbf{1 5}$ & $\mathbf{5 . 7 \%}$ \\
Consumo perjudicial & 1 & 0.4 & 1 & 0.4 & $\mathbf{2}$ & $\mathbf{0 . 8 \%}$ \\
Dependencia al alcohol & 1 & 0.4 & 1 & 0.4 & $\mathbf{2}$ & $\mathbf{0 . 8 \%}$ \\
\hline & Total & $\mathbf{3 7}$ & $\mathbf{1 4 \%}$ & $\mathbf{2 2 9}$ & $\mathbf{8 6 \%}$ & $\mathbf{2 6 6}$ & $\mathbf{1 0 0 \%}$ \\
\hline
\end{tabular}

Nota: Fuente: Cuestionario de Identificación de los Trastornos debidos al Consumo de Alcohol (AUDIT), (OMS, 2001), cédula de identificación de datos, $\mathbf{C M =}$ Conocimiento medio, $\mathbf{C A}=$ Conocimiento alto, $n=266$.

Para responder el consumo de alcohol sobre el conocimiento de COVID-19, se integró la Tabla 4, obteniendo los siguientes resultados: 32.2\% de los participantes que tiene un conocimiento alto no consumen alcohol, el $47.7 \%$ lo hacen de manera ocasional, $5.3 \%$ cuentan con una ingesta de riesgo y el $0.8 \%$ restante bebe de manera perjudicial o tiene dependencia a esta droga (0.4\% para cada una). Por otro lado, el $7.5 \%$ de los que no consumen alcohol tienen un conocimiento medio de la nueva enfermedad, $5.3 \%$ beben de manera ocasional y al menos el $0.4 \%$ la ingieren de una forma riesgosa, al igual que los participantes que toman de modo perjudicial o cuentan con una adicción. 
Tabla 5. Clasificación de la COVID-19 y consumo de tabaco

\begin{tabular}{|c|c|c|c|c|c|c|}
\hline \multirow[t]{3}{*}{ Consumo de tabaco } & \multicolumn{6}{|c|}{ Conocimiento de COVID-19 } \\
\hline & \multicolumn{2}{|c|}{$\mathbf{C M}$} & \multicolumn{2}{|c|}{ CA } & \multicolumn{2}{|c|}{ Total } \\
\hline & $\mathbf{f}$ & $\%$ & $\mathbf{f}$ & $\%$ & $\mathbf{f}$ & $\%$ \\
\hline No consume & 18 & 6.8 & 93 & 35.0 & 111 & $41.8 \%$ \\
\hline Riesgo bajo & 9 & 3.4 & 70 & 26.1 & 79 & $29.5 \%$ \\
\hline Riesgo moderado & 10 & 3.8 & 64 & 24.1 & 74 & $27.9 \%$ \\
\hline Riesgo alto & 0 & 0 & 2 & 0.8 & 2 & $0.8 \%$ \\
\hline Total & 37 & $14 \%$ & 229 & $86 \%$ & 266 & $100 \%$ \\
\hline
\end{tabular}

Nota: Fuente: Prueba de Detección de Consumo de Alcohol, Tabaco y Sustancias (ASSIST), (OMS, 2011), cédula de identificación de datos, $\mathbf{C M}=$ Conocimiento medio, $\mathbf{C A}=$ Conocimiento alto, $n=266$.

Para contrastar el conocimiento de los participantes en relación con el COVID-19 y su consumo de tabaco se realizó la Tabla 5, destacando que el $35.0 \%$ de los que tienen un alto conocimiento de esta afección, no son consumidores, $26.1 \%$ tiene un riesgo bajo, $24.1 \%$ lo hacen de manera moderada y al menos un $0.8 \%$ fuman peligrosamente. De forma similar, se encuentran los usuarios con conocimiento medio ya que el $6.8 \%$ no inhalan cigarros, $3.4 \%$ lo hacen de menor forma llegando a un riesgo bajo y al menos el $3.8 \%$ lo hace de manera moderada.

Tabla 6. Prueba de normalidad de variables

\begin{tabular}{lccc}
\hline \multirow{2}{*}{ Variables } & \multicolumn{3}{c}{ Kolmogorov Smirnov } \\
\cline { 2 - 4 } & Estadístico & gl & Sig. \\
\hline Conocimiento del COVID-19 & .136 & 266 & $\mathbf{. 0 0 0}$ \\
Consumo de alcohol & .272 & 266 & $\mathbf{. 0 0 0}$ \\
Consumo de tabaco & .280 & 266 & $\mathbf{. 0 0 0}$ \\
\hline
\end{tabular}

Nota: Fuente: Cuestionario de Identificación de los Trastornos debidos al Consumo de Alcohol (AUDIT), (OMS, 2001), Prueba de Detección de Consumo de Alcohol, Tabaco y Sustancias (ASSIST), (OMS, 2011), *Sig. $<0.05$ (variable no normal), $n=266$.

Para evaluar la normalidad de las variables en estudio, se utilizó la prueba estadística de Kolmogorov Smirnov (García, González \& Jornet, 2010), que se aplica para determinar el comportamiento de las mismas cuando se tiene una población mayor a 60 casos (Celis \& Labrada, 2014), dado el resultado que no existe agrupación unificada, se rechaza el criterio normal, puesto que se obtuvo un puntaje de significancia inferior (Sig. $<0.05$ ), por ello se determina utilizar la prueba de correlación de Rho Spearman (Díaz et al., 2014), por no tener homocedasticidad (Tabla 6).

Para establecer la relación de variables (Tabla 7), se utilizó la prueba estadística Rho Spearman (34), dado el valor no normal. Quienes tuvieron conocimiento alto del COVID-19 (C19), fueron los de generaciones avanzadas 
(GDP), es decir, existe mayor compromiso a la formación de temas actuales, realizando búsqueda de su propio autoaprendizaje, puesto que el problema es reciente para todos $\left(\mathrm{r}_{\mathrm{s}}=.186, \mathrm{p}<0.01\right)$, elementos que se respalda al evaluar la edad (GEP), dado que sobresalieron los de 21 a 23 años ( $\mathrm{r}_{\mathrm{s}}=.124$, $\left.\mathrm{p}<0.05\right)$, y son estos, quien mayor permanencia han tenido en la Facultad de Enfermería y han cursado Experiencias Educativas y semestres (SCA) que les otorgaron herramientas básicas e importancia del cuidado de la persona sana o enferma $\left(\mathrm{r}_{\mathrm{s}}=.184, \mathrm{p}<0.01\right)$. Por otro lado, la Generación del participante (GDP), es acorde al grupo de edad (GEP), $\left(\mathrm{r}_{\mathrm{s}}=.578, \mathrm{p}<0.01\right)$, semestre (SCA) $\left(\mathrm{r}_{\mathrm{s}}=.993\right.$, $\mathrm{p}<0.01)$ y son quienes viven con sus padres (ADV), $\left(\mathrm{r}_{\mathrm{s}}=.174, \mathrm{p}<0.01\right)$, es decir, tienen la protección de hogar, alimento, ropa y cobertura de necesidades básicas como seres humanos. En cuanto a las personas que tuvieron diagnóstico de COVID-19 (T19), tuvo que guardar cuarentena en casa (CPC), para proteger la salud $\left(\mathrm{r}_{\mathrm{s}}=.785, \mathrm{p}<0.01\right)$, por esto, es de suma importancia vigilar al grupo vulnerable pues están en riesgo de re infección o tener complicaciones al estado de salud, asimismo, se debe considerar importante la formación de los estudiantes en la temática actual para que éstos al mismo tiempo, informen a sus familias.

Por otra parte, el nivel de conocimiento bajo del COVID-19 (C19), determinó el consumo de alcohol (CAL), es decir, que en aquellas personas que tuvieron resultados bajos, aumentó el uso de la sustancia, sin medir las consecuencias al estado de salud $\left(\mathrm{r}_{\mathrm{s}}=-.121, \mathrm{p}<0.05\right)$. En cuanto al consumo de alcohol (CAL), quienes puntuaron como ocasional, se clasificaron en usuarios de riesgo bajo, moderado y alto (CTA) y permaneció la utilización de ambas sustancias, es decir que a mayor presencia de una, se agudizó la otra $\left(\mathrm{r}_{\mathrm{s}}=.138, \mathrm{p}<0.01\right)$, por tanto, puede ser un elemento principal para el diseño de actividades que se enfoquen en disminuir el problema, puesto que se encuentran en formación, personas que estarán dedicadas al cuidado de salud, máxime que es en la familia donde se aprueba la utilización de alcohol (ADV), es decir, al no percibir el riesgo mayor presencia de éste $\left(\mathrm{r}_{\mathrm{s}}=.139, \mathrm{p}<0.05\right)$. 
Tabla 7. Correlación de variables mediante la prueba de Rho Spearman

\begin{tabular}{|c|c|c|c|c|c|c|c|c|c|c|}
\hline & Variables & C19 & CAL & CTA & GDP & GEP & ADV & SCA & T19 & СРC \\
\hline C19 & $\begin{array}{l}\text { CC } \\
\text { Sig. (bilateral) } \\
\mathrm{N}\end{array}$ & $\begin{array}{r}1.000 \\
26 \dot{6}\end{array}$ & & & & & & & & \\
\hline CAL & $\begin{array}{l}\mathrm{CC} \\
\text { Sig. (bilateral) } \\
\mathrm{N}\end{array}$ & $\begin{array}{r}-.121 * \\
.049 \\
266\end{array}$ & $\begin{array}{r}1.00 \\
266\end{array}$ & & & & & & & \\
\hline CTA & $\begin{array}{l}\mathrm{CC} \\
\text { Sig. (bilateral) } \\
\mathrm{N}\end{array}$ & $\begin{array}{r}-.096 \\
.117 \\
266\end{array}$ & $\begin{array}{r}.138 * \\
.025 \\
266\end{array}$ & $\begin{array}{r}1.000 \\
266\end{array}$ & & & & & & \\
\hline GDP & $\begin{array}{l}\mathrm{CC} \\
\text { Sig. (bilateral) } \\
\mathrm{N}\end{array}$ & $\begin{array}{r}.186 * * \\
.002 \\
266\end{array}$ & $\begin{array}{r}-.109 \\
.075 \\
266\end{array}$ & $\begin{array}{r}-.101 \\
.101 \\
266\end{array}$ & $\begin{array}{r}1.000 \\
266\end{array}$ & & & & & \\
\hline GEP & $\begin{array}{l}\mathrm{CC} \\
\text { Sig. (bilateral) } \\
\mathrm{N}\end{array}$ & $\begin{array}{r}.124 * \\
.043 \\
266\end{array}$ & $\begin{array}{r}-.072 \\
.241 \\
266\end{array}$ & $\begin{array}{r}-.058 \\
.342 \\
266\end{array}$ & $\begin{array}{r}.578 * * \\
.000 \\
266\end{array}$ & $\begin{array}{r}1.000 \\
266\end{array}$ & & & & \\
\hline ADV & $\begin{array}{l}\mathrm{CC} \\
\text { Sig. (bilateral) } \\
\mathrm{N}\end{array}$ & $\begin{array}{l}.025 \\
.688 \\
266\end{array}$ & $\begin{array}{r}.139 * \\
.023 \\
266\end{array}$ & $\begin{array}{l}.010 \\
.872 \\
266\end{array}$ & $\begin{array}{r}.174 * * \\
.004 \\
266\end{array}$ & $\begin{array}{r}.303 * * \\
.000 \\
266\end{array}$ & $\begin{array}{r}1.000 \\
266\end{array}$ & & & \\
\hline SCA & $\begin{array}{l}\mathrm{CC} \\
\text { Sig. (bilateral) } \\
\mathrm{N}\end{array}$ & $\begin{array}{r}.184 * * \\
.003 \\
266\end{array}$ & $\begin{array}{r}-.120 \\
.051 \\
266\end{array}$ & $\begin{array}{r}-.105 \\
.088 \\
266\end{array}$ & $\begin{array}{r}.993 * * \\
.000 \\
266\end{array}$ & $\begin{array}{r}.579 * * \\
.000 \\
266\end{array}$ & $\begin{array}{r}.178 * * \\
.004 \\
266\end{array}$ & $\begin{array}{r}1.000 \\
266\end{array}$ & & \\
\hline T19 & $\begin{array}{l}\mathrm{CC} \\
\text { Sig. (bilateral) } \\
\mathrm{N}\end{array}$ & $\begin{array}{r}-.061 \\
.325 \\
266\end{array}$ & $\begin{array}{r}-.033 \\
.595 \\
266\end{array}$ & $\begin{array}{r}-.089 \\
.146 \\
266\end{array}$ & $\begin{array}{r}-.099 \\
.108 \\
266\end{array}$ & $\begin{array}{r}-.105 \\
.088 \\
266\end{array}$ & $\begin{array}{r}-.038 \\
.542 \\
266\end{array}$ & $\begin{array}{r}-.101 \\
.099 \\
266\end{array}$ & $\begin{array}{r}1.000 \\
266\end{array}$ & \\
\hline СPC & $\begin{array}{l}\text { CC } \\
\text { Sig. (bilateral) } \\
\text { N }\end{array}$ & $\begin{array}{r}-.011 \\
.852 \\
266\end{array}$ & $\begin{array}{l}.030 \\
.623 \\
266\end{array}$ & $\begin{array}{r}-.065 \\
.294 \\
266\end{array}$ & $\begin{array}{r}-.072 \\
.245 \\
266\end{array}$ & $\begin{array}{r}-.043 \\
.489 \\
266\end{array}$ & $\begin{array}{r}-.021 \\
.734 \\
266\end{array}$ & $\begin{array}{r}-.075 \\
.224 \\
266\end{array}$ & $\begin{array}{r}.785^{* *} \\
.000 \\
266\end{array}$ & $\begin{array}{r}1.000 \\
266\end{array}$ \\
\hline
\end{tabular}

Nota: Fuente: cédula de identificación de datos, Cuestionario de Identificación de los Trastornos debidos al Consumo de Alcohol (AUDIT), (Organización Mundial de la Salud, 2001), Prueba de Detección de Consumo de Alcohol, Tabaco y Sustancias (ASSIST), (Organización Mundial de la Salud, 2011), C19= Conocimiento de COVID-19, CAL= Consumo de alcohol, CTA= Consumo de tabaco, GDP= Generación del participante, GEP= Grupo de edad del participante, $\mathbf{A D V}=$ Actualmente, en dónde vive, $\mathbf{S C A}=$ Semestre que cursa actualmente, T19= Usted tiene o tuvo diagnóstico de COVID-19, $\mathbf{C P C}=$ Estuvo en cuarentena por probable diagnóstico o diagnóstico confirmado por COVID-19, CC= Coeficiente de correlación, $\mathbf{N}=$ Número de casos, * La correlación es significativa al nivel 0.05 (bilateral), **. La correlación es significativa al nivel 0.01 (bilateral), $n=266$.

\section{Discusión}

Sun et al. (2020), Cunial et al. (2020), Vieitez et al. (2020), Aguilar, Lazarevich, Mendoza \& López, (2018), REVIVA (2017) y Betancourth, Tacan \& Córdoba (2017), destacaron la participación de las mujeres puesto que todos reportaron más del $50.0 \%$, coincidiendo con esta investigación donde prevalece el mismo género, así como el grupo de edad con una media 
de 21 a 23 años. Ahora bien, conforme al estado civil de igual forma se detecta semejanza con las investigaciones de Aguilar, Lazarevich, Mendoza \& López (2018) REVIVA (2017) y Betancourth, Tacan \& Córdoba (2017), ya que representan el $88.3 \%, 94.1 \%$ y $93.8 \%$ como usuarios solteros respectivamente. Sin embargo, datos relacionados con escolaridad (generación y semestre que cursan), religión, lugar de origen y donde viven los usuarios no se detectaron, encontrando desigualdad con este trabajo a excepción de Aguilar, Lazarevich, Mendoza \& López (2018), donde únicamente reportan que 61.3\% de los casos vivían en casa de sus progenitores asemejándose al porcentaje obtenido $(77.7 \%)$.

Por otro lado, al analizar el consumo de alcohol se reportan similitudes y discrepancias con los demás trabajos. Para el estudio de Sun et al. (2020), existe una diferencia en el rango de frecuencia puesto que en la población que estudiaron, $18.7 \%$ beben de manera ocasional en comparación con el 53\% reportado en este proyecto al igual que los que ingieren regularmente existiendo $26.4 \%$ de diferencia y los que no toman bebidas alcohólicas (3.4\% contra $39.7 \%$ ).

De la misma manera, existen discrepancia con los resultados obtenidos por Cunial et a. (2020), puesto que refieren que tan solo el $15.95 \%$ no toma bebidas embriagantes en comparación con el $36.5 \%$ reportado en este trabajo, $15.5 \%$ bebía todos los días en comparación y $4.79 \%$ lo hacía sólo fines de semana en comparación con $0.8 \%$ de los usuarios que lo hace de dos a cuatro veces por semana. Sin embargo, existe similitud en el consumo mensual y semanal, puesto que solo existe una diferencia de $1.06 \%$. Por otro lado, se identificó similitud con el estudio de Aguilar, Lazarevich, Mendoza \& López (2018), donde la mayoría ingirió alcohol (95.0\%) en el último mes (66.2\%) y durante el último año, provocaron lesiones por este hábito con $4.0 \%$.

Por otra parte, con el proyecto de Martínez et al. (2018), únicamente se encontraron diferencias con los resultados, pues refieren que el $92.0 \%$ de los encuestados bebió alguna vez en su vida, $90.2 \%$ lo seguían haciendo en el último año, $38.7 \%$ de ellos consumen en forma de riesgo y $9.4 \%$ lo hizo en los últimos 30 días, semejándose con lo obtenido por Betancourth et al. (2017). No obstante, al analizar los datos obtenidos en el estudio Percibite 2 (REVIVA, 2017), destacaron los valores de ingesta de alcohol de por vida y consumo en los últimos 12 meses, existiendo $8.9 \%$ y $6.4 \%$ de diferencia con lo obtenido en este trabajo de investigación con lo que existe similitud en estas variables mencionadas. Por otro lado, respecto al consumo de tabaco se detectaron diferencias en algunos estudios de Sun et al. 2020 y Martínez et al. (2018), puesto que más alumnos han probado tabaco (59.0\%), 58.2\% fuma actualmente, $53.0 \%$ tiene mayor prevalencia de consumo ocasional y $25.2 \%$ ha empezado a consumir tabaco (últimos 3 meses). 
Sin embargo, para el apartado que evalúa conocimiento sobre el COVID-19 se identificó similitud entre las respuestas obtenidas que el 5.5\%, consideraron que su salud puede empeorar si se enferman (de COVID-19), el $0.6 \%$ reconoce los síntomas básicos y el $18.5 \%$ sabe que medidas se deben seguir para prevenir la enfermedad, siendo ésta una diferencia entre ambos proyectos, independientemente de que Vieitez et al. (2020), no clasificaron el nivel de conocimiento de las personas.

De esta manera, son evidentes las diferencias entre los valores de consumo de alcohol y tabaco que se encontraron en los trabajos, independientemente de las igualdades en el rango de edad, estado civil y género que presentan los usuarios. Para concluir, no existieron datos que relacionaran el consumo de alcohol y tabaco con el conocimiento o cuarentena por la nueva afección, debido a que en la actualidad no hay suficientes investigaciones que se enfoquen en estudiar la relación de estas variables entre sí pues aún se desconoce mucha información referente al origen, factores y causa del COVID-19.

\section{Conclusiones}

Para concluir la investigación y los aportes hacia el conocimiento se señala que, en cuanto a los datos sociodemográficos de la población en estudio; la muestra fue de 266 estudiantes inscritos en la Facultad de Enfermería Orizaba de los cuales el $79.6 \%$ son mujeres y $20.4 \%$ hombres con rango de edad de 21 a 23, cursando el séptimo semestre. El 88.7\% son solteros y provienen de zonas urbanas $(80.8 \%)$ y profesan el catolicismo $(77.7 \%)$, el 92.5\% no presentó diagnóstico confirmado de COVID-19 y $88.3 \%$ se resguardó en casa a pesar de no haber padecido esta afección. Lo anterior indica que los profesionales de enfermería que se insertarán a laborar en las diferentes instituciones del país de la región y el país son jóvenes en mayor proporción mujeres, con una formación integral para atender las demandas de salud de la población, fundamentada en conocimientos, habilidades, actitudes y valores éticos en el cuidado y colaboración con el equipo de trabajo, acorde a las necesidades y promoviendo la salud como lo expresa González et al. (2014).

Para analizar el nivel de conocimiento que tiene la población en estudio sobre la cuarentena por COVID-19, se examinaron diferentes dimensiones, el primero (conocimiento general) obtuvo un rango mayor de respuestas correctas siendo $82.7 \%$, lo que significa que la mayoría reconoce el nombre del nuevo coronavirus y la enfermedad que causa, $99.2 \%$ conoce el método de propagación y el $100 \%$ identifica que los virus mencionados causan enfermedades en seres humanos y animales.

En la clasificación del consumo de alcohol el 39.8\% de los estudiantes no beben sustancias embriagantes, $53.0 \%$ lo hacen de manera ocasional, 5.6\% 
de riesgo y $0.8 \%$ perjudicial o tienen dependencia a esta droga, mientras que para el tabaco el $41.7 \%$ no inhala esta droga, $29.7 \%$ tiene riesgo bajo, $27.8 \%$ moderada y al menos $0.8 \%$ fuma a tales grados de padecer un riesgo alto para su salud, es decir, que más de la mitad de la muestra, tiene un comportamiento adictivo, por tanto, es de gran importancia la continuidad de estudios e intervenciones a fin de regular el patrón hacia drogas legales, ya que estos, pueden potenciar riesgo o complicaciones y de no hacerlo, el nivel de calidad de cuidados, podría ser deficiente y por tanto, exponer a la población receptora de atención o los sistemas de salud.

Para determinar si la cuarentena por COVID-19, es un factor asociado al consumo de drogas legales en estudiantes de Enfermería, por el valor de la prueba de correlación ( $\mathrm{rs}=-.121, \mathrm{p}<0.05$ ), se confirma la hipótesis de trabajo, dando respuesta a la pregunta de investigación: la cuarentena por COVID-19 es un factor asociado al consumo de drogas legales en estudiantes de Enfermería a pesar de que la mayoría se resguardó en casa y no presentó diagnóstico confirmado e incluso, se puede observar que al beber líquidos embriagantes es más probable utilizar tabaco ( $r s=.138, \mathrm{p}<0.01$ ), como complemento, a pesar de que la mayoría de los estudiantes vivan en casa con sus padres $(\mathrm{rs}=.139, \mathrm{p}<0.05)$.

Por tanto, se debe considerar importante, establecer estrategias de atención y educación en los estudiantes, ya que su tiempo libre o para disminuir la tensión que provoca la cuarentena por COVID-19, lo utilizan para el uso de drogas legales y esto, puede agudizar el problema. Ante esto, se recomienda que los profesionales de salud, así como estudiantes de Enfermería sigan realizando trabajos de investigación con objetivos similares a los mencionados en este proyecto, con enfoques cuantitativos, cualitativos o mixtos para servir como referencias empíricas e incluso inspirar a la realización de programas de salud que ayuden a prevenir o reducir las consecuencias psicológicas de las pandemias que en la actualidad son un problema de salud pública.

Además, se deben formar a los profesores en el manejo de técnicas psicológicas para el manejo de estrés como señala Tenelanda et al. (2019) y a los padres de familia en la atención de jóvenes y adolescentes para la prevención y educación en salud en el consumo de drogas legales y con el uso de estrategias efectivas, implementarlas en este período de cuarentena por COVID-19, proponiendo el uso eficiente del tiempo libre y actividades que disminuyan el estrés, preocupación y tensión que puedan vivir en este período de pandemia y que conduzca al uso de alcohol y tabaco, evitando así como lo expresa Christiansen (2016), la narcoviolencia que puede traer grandes problemas a la salud si no es considerada como un problema que puede afectar no solo a quien lo consume, sino a quien está dependiente de los cuidados profesionales. 
Así también, las instituciones de educación superior tienen el desafío de dotar los recursos tecnológicos a los profesores y estudiantes (Méndez et al., 2020) que permita el desarrollo de nuevas propuestas educativas desde espacios virtuales y procesos que permitan continuar con la calidad educativa donde integren saberes enfocados al consumo de drogas legales y el período de pandemia.

\section{References:}

1. Aguilar, J., Lazarevich, I., Mendoza, J., \& López, F. (noviembre, 2018). Consumo de alcohol en estudiantes universitarios mexicanos del área de salud. Revista de Ciencias Clínicas, 20(1). Recuperado de https://www.terceridad.net/publicaciones/Uam191202Aguilar\%20PDF.pdf.pdf

2. Arceo, J., Ornelas, J., \& Domínguez, S. (2010). Manual de medicina basada en evidencias. México: El Manual Moderno.

3. Babor, T., Higgins, J., Saunders, J., \& Monteiro, M. (2001). The Alcohol Use Disorders Identification Test Guidelines for Use in Primary Care. Recuperado de http://whqlibdoc.who.int/hq/2001/who_msd_msb_01.6a.pdf

4. BBC News. (11 de febrero de 2020a). Enfermedad por coronavirus llamada Covid-19. Recuperado de https://www.bbc.com/news/worldasia-china-51466362

5. BBC News. (17 de marzo de 2020b). Coronavirus: qué es el distanciamiento social y por qué los expertos insisten en practicarlo para combatir la pandemia de covid-19. Recuperado de https://www.bbc.com/mundo/noticias-51899782

6. Betancourth, S., Tacan, B., \& Córdoba, E. (marzo, 2017). Consumo de alcohol en estudiantes universitarios colombianos. De Universidad y Salud, 19(1). Recuperado de http://www.scielo.org.co/pdf/reus/v19n1/0124-7107-reus-19-0100037.pdf

7. Canales, F., Alvarado, E., \& Pineda, E. (2013). Metodología de la investigación; Manual para el desarrollo de personal de salud. México: Limusa.

8. Celis, A., \& Labrada, V. (2014). Bioestadística ( $3^{a}$ Ed.). México: El Manual Moderno. México.

9. Centers for Disease Control and Prevention [CDC]. (2020). Quarantine and isolation. Recuperado de https://www.cdc.gov/quarantine/quarantineisolation.html

10. Comisión Nacional contra las Adicciones [CONADIC]. (2017). Encuesta Nacional de Consumo de Drogas, Alcohol y Tabaco, ENCODAT 2016-2017. Recuperado de 
https://www.gob.mx/salud\%7Cconadic/acciones-yprogramas/encuesta-nacional-de-consumo-de-drogas-alcohol-ytabaco-encodat-2016-2017-136758

11. Cunial, S., Dulbecco, P., \& Güelman, M. (2020). Consumo de alcohol en la cuarentena por covid-19. Recuperado de https://www.issup.net/files/2020-06/Estudio-alcohol-encuarentena\%20G.GERMANI.pdf

12. Christiansen, M. L. (2016). ¿Narcoinfierno O Narcolandia? Una Epistemología Intempestiva Sobre El Relato Oficial De La Violencia En México. European Scientific Journal, ESJ, 12(11), 129. https://doi.org/10.19044/esj.2016.v12n11p129

13. Díaz, I., García, C., León, M., Ruíz, F., Lizama, P., \& Boccardo, G. (2014). Guía de asociación entre variables (Pearson y Spearman en SPSS).

Recuperado de file:///C:/Users/enfermería/Downloads/9_Coeficientes_de_asociaci_n _Pearson_y_Spearman_en_SPSS.pdf.

14. García, M., Novalbos, J., Martínez, J., \& O’Ferral, C. (julio, 2016). Validación del test para la identificación de trastornos por uso de alcohol en población universitaria: AUDIT y AUDIT-C. Adicciones, 28(4).

Recuperado de http://www.adicciones.es/index.php/adicciones/article/view/775/0

15. García, R., González, J., \& Jornet, M. (2010). SPSS: pruebas no paramétricas: Kolmogorov Smirnov. Recuperado de https://www.uv.es/innomide/spss/SPSS/SPSS_0802A.pdf

16. Gómez, T., Diéguez, R., Pérez, M., Tamayo, O., \& Iparraguirre, A. (mayo, 2020). Evaluación del nivel de conocimiento sobre COVID-19 durante la pesquisa en la población de un consultorio. Revista estudiantil 16 de abril, 59(277). Recuperado de http://www.rev16deabril.sld.cu/index.php/16_04/article/view/925

17. González, E., \& Arras, A. M. (2014). Profesionalización de la enfermería y desempeño, desde la óptica del jefe inmediato superior en chihuahua, chihuahua. European Scientific Journal, ESJ, 10(1). https://doi.org/10.19044/esj.2014.v10n1p\%p. Recuperado de https://eujournal.org/index.php/esj/article/view/2564

18. Google (2020a). Crear y calificar cuestionarios con Formularios de Google. Recuperado

de https://support.google.com/docs/answer/7032287?hl=es-419

19. Google. (2020b). Instrumento de investigación cuarentena por COVID-19, factor asociado al consumo de drogas legales en estudiantes de Enfermería. Recuperado de https://forms.gle/yfiHUjKeNsfFCfbTA 
20. Grove, S., Gray, J., \& Burns, N. (2016). Investigación en enfermería; Desarrollo de la práctica de enfermería basada en la evidencia $\left(6^{a}\right.$ $E d$.). Barcelona, España: ELSEVIER.

21. H. Ayuntamiento de Orizaba. (2020). Comunicado Oficial 174, plan COVID-19 México. Recuperado de http://www.orizaba.gob.mx/covid19

22. Hernández, R., Fernández, C., \& Baptista, P. (2014). Metodología de la investigación $\left(6^{a} E d\right.$.). México D.F., México: Interamericana editores, S. A. de C. V.

23. Martínez, D., Martínez, F., \& Velázquez, A. (enero, 2018). Consumo de alcohol y tabaco en estudiantes de primero de Medicina de la Universidad del País Vasco. Revista española de drogodependencias, 43(1). Recuperado de https://www.aesed.com/upload/files/vol43/n1/num-completos/v43n1_orig1.pdf

24. Méndez, M. O., Olvera, M. Y., Cabrera, A. P., Ordoñez, J. E., Buendía, D. I., \& Guilcapi, M. A. (2020). Evaluación de la Virtualización del Aprendizaje y los Desafíos en la Educación Superior en el Instituto Superior Tecnológico Juan Bautista Aguirre. European Scientific Journal, ESJ, $16(31), \quad 81$. https://doi.org/10.19044/esj.2020.v16n31p81

25. Natera, G., Medina, P., López, S., Arroyo, M., Sánchez, Y., Pérez, A., Bautista, N., Hernández, S., \& Martínez, N. (2020). La covid, el alcohol y la depresión: ¿qué pasó en México en el primer nivel de atención y las DIT, durante la pandemia?. De la Organización Panamericana de la Salud. Recuperado de https://www.paho.org/es/documentos/covid-alcohol-depresion-quepaso-mexico-primer-nivel-atencion-dit-durante-pandemia

26. Orellana, L. (2001). Estadística descriptiva. Recuperado de http://www.dm.uba.ar/materias/estadistica_Q/2011/1/modulo\%20des criptiva.pdf

27. Organización Mundial de la Salud [OMS]. (2001). AUDIT. Cuestionario de identificación de los Trastornos debidos al Consumo de Alcohol. Pautas para su utilización en la Atención Primaria. Recuperado de https://www.paho.org/hq/dmdocuments/2009/audit_spa.pdf

28. Organización Mundial de la Salud [OMS]. (2004). Global status report on alcohol 2004. Recuperado de https://www.who.int/substance_abuse/publications/global_status_rep ort_2004_overview.pdf

29. Organización Mundial de la Salud [OMS]. (2011). La prueba de detección de consumo de alcohol, tabaco y sustancias (ASSIST), manual para uso en la atención primaria. Recuperado de 
https://apps.who.int/iris/bitstream/handle/10665/85403/97892753323 68_spa.pdf?sequence $=1$ \&isAllowed $=y$

30. Organización Mundial de la Salud [OMS]. (2020a). Consideraciones relativas a los ajustes de las medidas de salud pública y sociales en el contexto de la COVID-19. Orientaciones provisionales. Recuperado de https://apps.who.int/iris/bitstream/handle/10665/331970/WHO2019-nCoV-Adjusting_PH_measures-2020.1spa.pdf? sequence $=1 \&$ isAllowed $=y$

31. Organización Mundial de la Salud [OMS]. (2020b). Preguntas y respuestas sobre la enfermedad por coronavirus (COVID-19). Recuperado de https://www.who.int/es/emergencies/diseases/novelcoronavirus-2019/advice-for-public/q-acoronaviruses?gclid=Cj0KCQjw4f35BRDBARIsAPePBHxcsk59DsS xGo-N-

JiWpH4ayxAg_fsPJrg7Pe9VxjQ2P9QH8JglWboaAufQEALw_wcB

32. Organización Mundial de la Salud [OMS]. (2020c). Preguntas y respuestas sobre la enfermedad por coronavirus (COVID-19). Recuperado de https://www.who.int/es/emergencies/diseases/novelcoronavirus-2019/question-and-answers-hub/q-a-detail/coronavirusdisease-covid-19

33. Organización Panamericana de la Salud [OPS]. (2020a). Las redes sociales y COVID-19: la contribución de BIREME. Recuperado de https://www.paho.org/bireme/index.php?option=com_content\&view $=$ article $\&$ id=479:redes-sociais-e-covid-19-a-contribuicao-dabireme \&Itemid $=183 \&$ lang=es

34. Organización Panamericana de la Salud [OPS]. (2020b). Salud mental y COVID-19. Recuperado de https://www.paho.org/es/salud-mentalcovid-19

35. Organización Mundial de la Salud [OMS]. (2021). Coronavirus. Recuperado de topics/coronavirus\#tab=tab_1

36. Ortiz, F. G., \& García M. (2014). Metodología de la investigación; El proceso y sus técnicas. México: LIMUSA.

37. Oteo, A. (2005). Tendencias en el consumo de drogas en la juventud española y efectos sobre su salud. Recuperado de http://www.injuve.es/sites/default/files/RJ84-11.pdf

38. Pineda, J., Lara, A., Rocha, E., Piña, I., \& Romero, R. (2016). Perfil de ingreso de los estudiantes de la Licenciatura en Enfermería en una Institución de Educación Superior. Recuperado de https://www.medigraphic.com/pdfs/enfermeriaimss/eim2018/eim181f.pdf 
39. Polit, D., \& Hungler, B. (2000). Investigación científica en Ciencias de la Salud $\left(6^{a} E d\right.$.). México D. F: Mc Graw-Hill interamericana.

40. Psicología y mente (2021). ¿Qué son las drogas legales y qué tipos existen?. Recuperado de https://psicologiaymente.com/drogas/drogaslegales

41. Red Veracruzana de Investigación en Adicciones [REVIVA]. (2017). Reporte Técnico PERCÍBETE 2. De Universidad Veracruzana. Recuperado https://www.uv.mx/mpicd/files/2018/10/ReporteTecnicoPercibete2017.pdf

42. Secretaría de Educación Pública [SEP]. (2019). Principales cifras del sistema educativo nacional 2018-2019. Recuperado de https://www.planeacion.sep.gob.mx/Doc/estadistica_e_indicadores/pr incipales_cifras/principales_cifras_2018_2019_bolsillo.pdf

43. Secretaría de Salud [SS]. (2017a). ENCODAT Encuesta Nacional de Consumo de Drogas, Alcohol y Tabaco 2016-2017 reporte de alcohol. Recuperado de https://drive.google.com/file/d/1rMlKaWy34GR51sEnBK2u2q_BDK9LA0e/view

44. Secretaría de Salud [SS]. (2017b). ENCODAT Encuesta Nacional de Consumo de Drogas, Alcohol y Tabaco 2016-2017 reporte de tabaco. Recuperado https://drive.google.com/file/d/1Iktptvdu2nsrSpMBMT4FdqBIk8gikz $7 \mathrm{q} / \mathrm{view}$

45. Secretaría de Salud [SS]. (2020). Comunicado técnico diario nuevo coronavirus en el mundo (COVID-19) (Informe No. 37). Recuperado de

https://www.gob.mx/cms/uploads/attachment/file/537793/Comunicad o_Tecnico_Diario_COVID-19_2020.02.27.pdf

46. SPSS, Inc. (2006). Guía breve de SPSS 15.0. Recuperado de http://www.um.es/ae/soloumu/pdfs/pdfs_manuales_spss/SPSS $\% 20 \mathrm{Br}$ ief\%20Guide\%2015.0.pdf

47. Sun, Y., Li, Y., Bao, Y., Meng, S., Sun, Y., Schumann, G., Kosten, T., Strang, J., Lu, L., \& Shi, J. (2020). Brief report: increased addictive internet and substance use behavior during the COVID-19 pandemic in China. Recuperado de https://doi.org/10.1111/ajad.13066

48. Tamayo, M. (2014). El proceso de la investigación científica. México: LIMUSA.

49. Tenelanda, D., Damián, D. L., Célleri, S. P., Castillo, M. P., \& Inca, N. M. (2019). Técnicas Psicológicas Para Manejar El Estrés Académico En El Proceso De Aprendizaje Del Idioma Inglés. 
European Scientific Journal, ESJ, 15(4), 421. https://doi.org/10.19044/esj.2019.v15n4p421

50. Tiburcio, M., Rosette, M., Natera, G., Martínez, A., Carreño, S., \& Pérez, D. (marzo, 2016). Validez y confiabilidad de la prueba de detección de consumo de alcohol, tabaco y sustancias (ASSIST) en estudiantes universitarios. Adicciones, 28(1). Recuperado de https://www.adicciones.es/index.php/adicciones/article/view/786/750

51. Universidad Veracruzana [UV]. (2013). Licenciatura en Enfermaría, Plan de Estudios 2013. Recuperado de https://www.uv.mx/orizaba/enfermeria/files/2016/06/PLAN-DEESTUDIOS-junio-16.pdf

52. Vieitez, I., Larrea, S., Romero, F., \& Cicolella, L. (2020). Conocimientos, actitudes y prácticas relacionadas a la pandemia del covid-19 en comunidades indígenas de Yucatán y Chiapas. Recuperado de https://knowledgecommons.popcouncil.org/cgi/viewcontent.cgi?articl e=2144\&context=departments_sbsr-pgy 